\title{
Joint Data Streaming and Sampling Techniques for Detection of Super Sources and Destinations
}

\author{
Qi (George) Zhao Abhishek Kumar Jun (Jim) Xu \\ College of Computing, Georgia Institute of Technology
}

\begin{abstract}
Detecting the sources or destinations that have communicated with a large number of distinct destinations or sources during a small time interval is an important problem in network measurement and security. Previous detection approaches are not able to deliver the desired accuracy at high link speeds (10 to $40 \mathrm{Gbps}$ ). In this work, we propose two novel algorithms that provide accurate and efficient solutions to this problem. Their designs are based on the insight that sampling and data streaming are often suitable for capturing different and complementary regions of the information spectrum, and a close collaboration between them is an excellent way to recover the complete information. Our first solution builds on the standard hash-based flow sampling algorithm. Its main innovation is that the sampled traffic is further filtered by a data streaming module which allows for much higher sampling rate and hence much higher accuracy. Our second solution is more sophisticated but offers higher accuracy. It combines the power of data streaming in efficiently estimating quantities associated with a given identity, and the power of sampling in collecting a list of candidate identities. The performance of both solutions are evaluated using both mathematical analysis and trace-driven experiments on real-world Internet traffic.
\end{abstract}

\section{Introduction}

Measurement of flow-level statistics, such as total active flow count, sizes and identities of large flows, per-flow traffic, and flow size distribution are essential for network management and security. Measuring such information on high-speed links (e.g., $10 \mathrm{Gbps}$ ) is challenging since the standard method of maintaining per-flow state (e.g., using a hash table) for tracking various flow statistics is prohibitively expensive. More specifically, at very high link speeds, updates to the per-flow state for each and every incoming packet would be feasible only through the use of very fast and expensive memory (typically SRAM), while the size of such state is very large [7] and hence too expensive to be held in SRAM. Recently, the techniques for approximately measuring such statistics using a much smaller state, based on a general methodology called network data streaming, have been used to solve some of the aforementioned problems $[5,6,12,11,22]$. The main idea in net- work data streaming is to use a small and fast memory to process each and every incoming packet in real-time. Since it is impractical to store all information in this small memory, the principle of data streaming is to maintain only the information most pertinent to the statistic to be measured.

In this work, we design data streaming algorithms that help detect super sources and destinations. A super source ${ }^{1}$ is a source that has a large fan-out (e.g., larger than a predefined threshold) defined as the number of distinct destinations it communicates with during a small time interval. The concepts of super destination and fan-in can be defined symmetrically. Our schemes in fact solve a strictly harder problem than making a binary decision of whether a source/destination is a super source/destination or not: They actually provide accurate estimates of the fanouts/fan-ins of potential super sources/destinations. In this work a source can be any combination of "source" fields from a packet header such as source IP address, source port number, or their combination, depending on target applications. Similarly, a destination can be any combination of the "destination" fields from a packet header. We refer to the source-destination pair of a packet as the flow label and use these two terms interchangeably in the rest of this paper.

The problem of detecting super sources and destinations arises in many applications of network monitoring and security. For example, port-scans probe for the existence of vulnerable services across the Internet by trying to connect to many different pairs of destination IP address and port number. This is clearly a type of super source under our definition. Similarly, in a DDoS (Distributed Denial of Service) attack, a large number of zombie hosts flood packets to a destination. Thus the problem of detecting the launch of DDoS attacks can be viewed as detecting a super destination. This problem also arises in detecting worm propagation and estimating their spreading rates. An infected host often propagates the worm to a large number of destinations, and can be viewed as a super source. Knowing its fan-out allows us to estimate the rate at which the worm may spread. Another possible instance lies in peer-to-peer and content distribution networks, where a few servers or peers might attract a larger number of requests (for content) than they can handle while most of others in the network are relatively idle. Being able to detect such "hot spots" (a type of super destination) in real-time helps bal- 
ance the workload and improve the overall performance of the network. A number of other variations of the above applications, such as detecting flash crowds [9] and reflector attacks [15], also motivate this problem.

Techniques proposed in the literature for solving this problem typically maintain per-flow state, and cannot scale to high link speeds of 10 or $40 \mathrm{Gbps}$. For example, to detect port-scans, the widely deployed Intrusion Detection System (IDS) Snort [19] maintains a hash table of the distinct source-destination pairs to count the destinations each source talks to. A similar technique is used in FlowScan [17] for detecting DDoS attacks. The inefficiency in such an approach stems from the fact that most of the source-destination pairs are not a part of port scans or DDoS attacks. Yet, they result in a large number of source-destination pairs that can be accommodated only in DRAM, which cannot support the high access rates required for updates at line speed. More recent work [20] has offered solutions based on hash-based flow sampling technique. However, its accuracy is limited due to the typically low sampling rate imposed by some inherent limitations of the hash-based flow sampling technique discussed later in Section 3. A more comprehensive survey of related work is provided in Section 7.

In this paper we propose two efficient and accurate data streaming algorithms for detecting the set of super sources by estimating the fan-outs of the collected sources. These algorithms can be easily adapted symmetrically for detecting the super destinations. Their designs are based on the insight that (flow) sampling and data streaming are often suitable for capturing different and complementary regions of the information spectrum, and a close collaboration between them is an excellent way to recover the complete information. This insight leads to two novel methodologies of combing the power of streaming and sampling, namely, "filtering after sampling" and "separation of counting and identity gathering". Our two solutions are built upon these two methodologies respectively.

Our first solution, referred to as the simple scheme, is based on the methodology of "filtering after sampling". It enhances the traditional hash-based flow sampling algorithm to approximately count the fan-outs of the sampled sources. As suggested by its name, the design of this solution is very simple. Its main innovation is that the sampled traffic is further filtered by a simple data streaming module (a bit array), which guarantees that at most one packet from each flow is processed. This allows for much higher sampling rate (hence much higher accuracy) than achievable with traditional hash-based flow sampling. Our second solution, referred to as the advanced scheme, is more sophisticated than the simple scheme but offers even higher accuracy. Its design is based on the methodology of "separation of counting and identity gathering", which combines the power of streaming in efficiently estimating quantities (e.g., fan-out) associated with a given identity, and the power of sampling in generating a list of candidate identities (e.g., sources). Through rigorous theoretical analysis and extensive trace-driven experiments on real-world Internet traffic, we demonstrate these two algorithms produce very accurate fan-out estimations.

We also extend our advanced scheme for detecting the sources that have large outstanding fan-outs, defined as the number of distinct destinations it has contacted but has not obtained acknowledgments (TCP ACK) from. This extension has several important applications. One example is that in port-scans, the probing packets, which target a large number of destinations, will receive acknowledgments from only a small percentage of them. Another example is distributed TCP SYN attacks. In this case, the victim's TCP acknowledgments (SYN/ACK packets) to a large number of hosts for completing the TCP handshake (the second step) are not acknowledged. Our evaluation on bidirectional traffic collected simultaneously on a link shows that our solution estimates outstanding fanout with high accuracy.

The rest of this paper is organized as follows. In the next section, we present our design methodologies and provide an overview of the proposed solutions. Sections 3 and 4 describe the design of the two schemes in detail respectively and provide a theoretical analysis of their complexity and accuracy. Section 5 presents an extension of our scheme for estimating outstanding fan-outs. We evaluate our solutions in Section 6 using packet header traces of real-world Internet traffic. We discuss the related work in Section 7 before concluding in Section 8.

\section{Overview of our schemes}

As we mentioned above, accurate measurement and monitoring in high speed networks are challenging because the traditional per-flow schemes cannot scale to high link speeds. As a stop-gap solution, packet sampling has been used to keep up with high link speeds. In packet sampling, a small fraction $p$ of traffic is sampled and processed. Since the sampled packets constitute a much lower volume than the original traffic, a per-flow table stored in relatively inexpensive DRAM can handle all the updates triggered by the sampled packets in real-time [14]. Thus we can typically obtain complete information contained in the sampled traffic. The statistics of the original traffic are then inferred from that of the sampled traffic by "inverting" the sampling process, i.e., by compensating for the effects of sampling. However the accuracy of such sampling-based estimations is usually low, because the error is scaled by $1 / p$ and $p$ is typically small (e.g., 1/500) to make the sampling operation computationally affordable $[4,11,8]$. In other words, although the sampling-based approach allows for $100 \%$ accurate digesting of information on sampled traffic, a large amount of information may be lost during the sampling 
process.

Network data streaming ${ }^{2}$ has begun to be recognized as a better alternative to sampling for measurement and monitoring of high-speed links [10]. Contrary to sampling, a network data streaming algorithm will process each and every packet passing through a high-speed link to glean the most important information for answering a specific type of query, using a small yet well-organized data structure. This data structure is small enough to be fit into fast (yet expensive) SRAM, allowing it to keep up with high link speeds. The challenge is to design this data structure in such a way that it encodes the information we need, for answering the query, in a succinct manner. Data streaming algorithms, if available, typically offer much more accurate estimations than sampling for measuring network flow statistics. This is because, intuitively the sampling throws away a large percentage of information up front, while data streaming, which processes each and every packet, is often able to retain most of the most important information inside a small SRAM module.

In our context of detecting super sources, however, both sampling and data streaming are valuable for capturing different and complementary regions of the information spectrum, and a close collaboration between them is used to recover the complete information. There are two parts of information that we would like to know in finding super sources: one is the identities (e.g., IP addresses) of the sources that may be super sources. The other is the fanout associated with each source identity. We observe that data streaming algorithms can encode the fan-outs of various sources into a very succinct data structure. Such a data structure, however, typically only provides a lookup interface. In other words, if we obtain a source identity through other means, we are able to look up the data structure to obtain its (approximate) fan-out, but the data structure itself cannot produce any identities and is undecodable without such identities being supplied externally. On the other hand, sampling is an excellent way of gathering source identities though it is not a great counting device as we described earlier.

The above observation leads to one of the two aforementioned design methodologies, i.e., separating identity gathering and counting. The idea is to use a streaming data structure as a counting device and use sampling to gather the identities of potential super sources. Then we look up the streaming data structure using the gathered identities to obtain the corresponding counts. This methodology is used in our advanced scheme that employs a 2-dimensional bit array as the counting device, in parallel with an identity gathering module that adopts an enhanced form of sampling. We show that our sampling module has vanishingly small probability of missing the identity of any actual super sources and the estimation module produces highly accurate estimates of the fan-out of the potential super sources.
This scheme is especially suitable for very high link speeds of $10 \mathrm{Gbps}$ and above. We describe this scheme in Section 4.

We also explore another way of combing sampling and streaming, i.e., "filtering after sampling". Its idea is to employ a data streaming module between the sampling operation and the final processing procedure to efficiently encode whether a flow has been seen before. A careful design of this module guarantees that at most one packet in each flow needs to be processed. This allows us to achieve much higher sampling rate and hence much higher accuracy than the traditional flow sampling scheme. This solution works very well for relatively lower link speeds (e.g., $10 \mathrm{Gbps}$ and below). We describe this scheme in detail in Section 3.

\section{The simple scheme}

In this section we present a relatively simple scheme for detecting super sources. It builds upon the traditional hashbased flow sampling technique but can achieve a much higher sampling rate, and hence more accurate estimation. We begin with a discussion of some limitations of the traditional hash-based sampling approach, and then describe our solution that alleviates these limitations. We also present an analysis of the complexity and accuracy of the scheme.

\subsection{Limitations of traditional hash-based flow sampling}

There are two generic sampling approaches for network measurement: packet sampling and flow sampling. In the former approach, each packet is sampled independently with a certain probability, while in the latter, the sampling decision is made at the granularity of flows (i.e., all packets belonging to sampled flows are sampled). In the following, we only consider flow sampling since packet sampling is not suitable for our context of detecting super sources. ${ }^{3}$

A traditional flow sampling algorithm that estimates the fan-outs of sources works as follows. The algorithm randomly samples a certain percentage (say $p$ ) of sourcedestination pairs using a hashing technique (described next). The fan-out of each source in the sampled pairs is counted and then scaled by $1 / p$ to obtain an estimate of the fan-out of the source in the original traffic (i.e., before sampling). This counting process is typically performed using a hash table that stores the fan-out values (after sampling) of all sources seen in the sampled traffic so far, and a newly sampled flow will increment the fan-out counter of the corresponding hash node (or trigger the creation of a new node). Since the estimation error is also scaled by $1 / p$, it is desirable to make the sampling rate $p$ as high as possible. However, we will show that, at high link speeds, the traditional hash-based flow sampling approach may prevent us from achieving high sampling rate needed for accurate estimation. 
Flow sampling is commonly implemented using a simple hashing technique [3] as follows. First a hash function $g$ that maps a flow label to a value uniformly distributed in $[0,1)$ is fixed. When a packet arrives, its flow label is hashed by $g$. Given a sampling probability $p$, the flow is sampled if and only if the hashing result is no more than $p$. Recall that the purpose of flow sampling is to reduce the amount of traffic that needs to be processed by the aforementioned hash table which performs the counting. Clearly, it is desirable that a hash table that runs slightly faster than $p$ times the link speed, can keep up with the incoming rate of the sampled traffic (with rate $p$ ). For example, we would like a hash table (in DRAM) that is able to process a packet in $400 \mathrm{~ns}$ to handle all traffic sampled from a link with 10 million packets per second (i.e., one packet arrival per $100 \mathrm{~ns}$ on the average) with slightly less than $25 \%$ sampling rate. Unfortunately, we cannot achieve this goal with the current hash-based flow sampling approach for the following reason.

With hash-based flow sampling, if a flow is sampled, all packets belonging to the flow need to be processed by the hash table. Internet traffic is very bursty in the sense that the packets belonging to a flow tend to arrive in bursts and do not interleave well with packets from other flows and is also known to exhibit the following characteristic [5]: a small number of elephant flows contain most of the overall traffic while the vast majority of the flows are small. If a few elephant flows are sampled, their packets could generate a long burst of sampled traffic that has much higher rate than that can be handled by the hash table ${ }^{4}$. Therefore, with hash-based flow sampling, the sampling rate $p$ has to be much smaller than the ratio between the operating speed of the hash table and the arrival rate of traffic, thus leading to large estimation errors as discussed before. In the following subsection, we present an efficient yet simple solution to this problem, allowing the sampling rate to reach or even well exceed this ratio.

In [20] the authors propose a one-level filtering algorithm which uses the hash-based flow sampling approach described above, in conjunction with a hash table for counting the fan-out values. It does not specify whether DRAM or SRAM will be used to implement the hash table. If DRAM were used, it will not be able to achieve a high sampling rate as discussed before. If SRAM were used, the memory cost is expected to be prohibitive when the sampling rate is high. This algorithm appears to be effective and accurate for monitoring lower link speeds, but cannot deliver a high estimation accuracy when operating at high link speeds such as 10Gbps (the target link speeds are not mentioned in [20]).

\subsection{Our scheme}

We design a filtering technique that completely solves the aforementioned problem. It allows the sampling rate to be

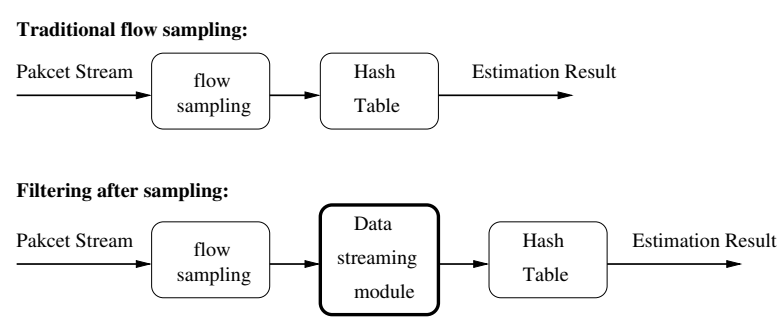

Figure 1: Traditional flow sampling vs. filtering after sampling

$$
\begin{aligned}
& \text { 1. Initialize } \\
& \text { 2. } G[r]:=0, \mathrm{r}=1,2, \ldots, w \\
& \text { 3. } / * w \text { is the size of the array } * / \\
& \text { 4. } u:=w \\
& \text { 5. } / * \text { variable } u \text { keep track the number of " } 0 \text { "s in } G * / \\
& \text { 6. Filtering after sampling } \\
& \text { 7. Upon each incoming sampled packet } p k t \\
& \text { 8. } \quad r:=h(<\text { pkt.src, pkt.dst }>) \\
& \text { 9. if } G[r]=0 \\
& \text { 10. } s:=\text { pkt.src } \\
& \text { 11. } \widehat{N_{s}}:=\widehat{N_{s}}+\frac{w}{u} \\
& \text { 12. } / * \text { The }\left(s, \widehat{N_{s}}\right) \text { pairs are maintained as a hash } \\
& \text { table } L . * / \\
& \text { 13. } G[r]:=1 \\
& \text { 14. } u:=u-1 \\
& \text { 15. } / * \text { The number of " } 0 \text { "s is decreased by } 1 * /
\end{aligned}
$$

Figure 2: Algorithm of updating data streaming module.

very close to the ratio between the hash table speed and the link speed in the worst-case and well exceed the ratio otherwise. Its conceptual design is shown in Figure 1. Compared with the traditional flow sampling approach, our approach places a data streaming module between the hashbased flow sampling module and the hash table (for counting). This streaming module guarantees that at most one packet from each sampled flow needs to be processed by the hash table. This will completely smooth out the aforementioned traffic bursts in the flow-sampled traffic, since such bursts are caused by highly bursty arrivals from one or a small number of elephant flows and now only the first packets of these flows may trigger updates to the hash table.

The data structure and algorithm of the data streaming module are shown in Figure 2. Its basic idea is to use a bit array $G$ to remember whether a flow label, a sourcedestination pair in our context, has been processed by the hash table. Let the size of the array be $w$ bits. We fix a hash function $h$ that maps a flow label to a value uniformly distributed in $[1, w]$. The array is initialized to all " 0 "s at the beginning of a measurement epoch. Upon the arrival of a packet $p k t$, we hash its flow label $(<p k t . s r c, p k t . d s t>)$ using $h$ and the result $r$ is treated as an index into the array 
$G$. If $G[r]$ is equal to 1 , our algorithm concludes that a packet with this flow label has been processed earlier, and takes no further action. Otherwise (i.e., $G[r]$ is 0 ), this flow label will be processed to update the corresponding counter $N_{\text {pkt.src }}$ maintained in a hash table $L$. Then $G[r]$ is set to 1 to remember the fact that a packet with this flow has been seen and processed. This method clearly ensures that at most one packet from each sampled flow is processed by $L$. However, due to hash collisions, some sampled flows may not be processed at all since their corresponding bits in $G$ would be set by their colliding counterparts. ${ }^{5}$ The update procedure of the hash table $L$, described next, statistically compensates for such collisions.

Now we explain our statistical estimator, which is the computation result of the hash table update procedure shown in Figure 2 (line 11). Suppose the number of " 0 " entries in $G$ (with size $w$ ) is $u$ right before a packet $p k t$ with source $s$ arrives ( $s:=p k t . s r c$ in line 10). Assume $p k t$ belongs to a new flow and its flow label hashes to an index $r$. The value of $G[r]$ has value 0 with probability $\frac{u}{w}$. Therefore to obtain an unbiased estimator $\widehat{N}_{s}$ of the fan-out of the source $s$ on the sampled traffic, we should statistically compensate for the fact that with probability $1-\frac{u}{w}$, the bit $G[r]$ has value 1 and $p k t$ will miss the update to $L$ due to aforementioned hash collisions. It is intuitive that if we add $\frac{w}{u}$ to $\widehat{N}_{s}$, the resulting estimator is unbiased. To be more precise, suppose in a measurement epoch, the hash table is updated by altogether $K$ packets $\left\{p k t_{j}, j=1,2, \ldots, K\right\}$ from a source $s$, whose flow labels hash to locations $r_{j}$ 's where $G\left[r_{j}\right]=0$, and there are $u_{j} 0$ 's in $G$ right before $p k t_{j}$ arrives, respectively. The output of the hash table $L$, which is an unbiased estimator of the fan-out of $s$ on the sampled traffic, is

$$
\widehat{N_{s}}=\sum_{j=1}^{K} \frac{w}{u_{j}}
$$

We show in the following lemma that this is an unbiased estimator of $N_{s}$ and its proof can be found in [24].

Lemma $1 \widehat{N}_{s}$ is an unbiased estimator of $N_{s}$, i.e., $E\left[\widehat{N_{s}}\right]=N_{s}$.

Then an unbiased estimator of the fan-out $F_{s}$ of source $s$ is given by scaling $\widehat{N}_{s}$ by $1 / p$, i.e.,

$$
\widehat{F_{s}}=\frac{1}{p} \sum_{j=1}^{K} \frac{w}{u_{j}}
$$

where $p$ is the sampling rate used in the flow sampling. We show in the following theorem that the estimator $\widehat{F_{s}}$ is unbiased. Its proof uses Lemma 1 and is also provided in [24].

Theorem $1 \widehat{F_{s}}$ is an unbiased estimator of $F_{s}$, i.e., $E\left[\widehat{F_{s}}\right]=F_{s}$.
We now demonstrate that this solution will completely smooth out the aforementioned problem of traffic bursts , and allow the sampling rate to be close to the ratio between the hash table speed and the link rate, the theoretical upper limit in the worst case. The worst case for our scheme is that each flow contains only one packet (e.g., in the case of DDoS attacks $)^{6}$. Even in this worst case, the update times to the hash table (viewed as a random process) is very close to Poisson ${ }^{7}$ (nonhomogeneous as the value of $u$ varies over time) since each new flow is sampled independently. Due to the "benign" nature of this arrival process, by employing a tiny SRAM buffer (e.g., holding 20 flow labels of 64 100 bits each), a hash table that operates slightly faster than the average rate of this process will only miss a negligible fraction of updates due to buffer overflow. This process can be faithfully modeled as a Markov chain for rigorous analysis. We elaborate it with a numerical example in [24] and omit it here due to lack of space.

Notice that in Figure 2 the variable $u$, the number of " 0 " entries in $G$, decreases as more and more sampled flows are processed. When more and more packets pass through the data streaming module, $u$ becomes small and hence the probability for a new flow to be recorded, $\frac{u}{w}$, decreases. Thereby the estimation error will increase. To maintain high accuracy, we specify a minimum value $u_{\min }$ for $u$. Once the value of $u$ drops below $u_{\min }$, the estimation procedure will use a new array (set to all " 0 "s initially) and start a new measurement epoch (with an empty hash table). Two sets of arrays and hash tables will be operated in an alternating manner so that the measurement can be performed without interruption. The parameter $u_{\min }$ is typically set to around $w / 2$ (i.e., "half full").

\subsection{Complexity analysis}

The above scheme has extremely low storage (SRAM) complexity and allows for very high streaming speed.

Memory (SRAM) consumption. Each processed flow only consumes a little more than one bit in SRAM. Thus a reasonable amount of SRAM can support very high link speeds. For example, assuming the average flow size of 10 packets [11], 512KB SRAM is enough to support a measurement epoch which is slightly longer than 2 seconds for a link with 10 million packets per second even without performing any flow sampling. With $25 \%$ flow sampling which is typically set for OC-192 links the SRAM requirement is even brought down to $128 \mathrm{~KB}^{8}$

Streaming speed. Our algorithm in Figure 2 has two branches to deal with the packets arriving at the data streaming module. If the corresponding bit is " 1 ", the packets only require one hash function computation and one read to SRAM. Otherwise they require one hash function computation, one read and one write (at the same location) to SRAM and an update to the hash table. Using efficient hardware implementation of hash function [18] and 5ns 
Packet stream

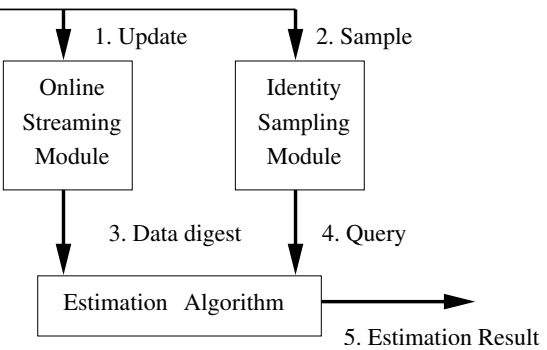

Figure 3: System model of the advanced scheme

SRAM, all operations in the data streaming module can be finished in 10's of $n s$ in both cases.

\subsection{Accuracy analysis}

Now we establish the following theorem to characterize the variance of the estimator $\widehat{F_{s}}$ in Formula 2 . Its proof can be found in [24]

\section{Theorem 2}

$$
\operatorname{Var}\left[\widehat{F_{s}}\right] \approx \frac{\sum_{j=1}^{p F_{s}} \frac{w-u_{j}}{u_{j}}}{p^{2}}+\frac{F_{s}(1-p)}{p}
$$

Remark: The above variance consists of two terms. The first term corresponds to the variance of the error term in estimating the sampled fan-out, scaled by $\frac{1}{p^{2}}$ (to compensate for sampling), and the second term corresponds to the variance of the error term in inverting flow sampling process. Since these two errors are approximately orthogonal to each other, their total variance is the sum of their individual variances.

\section{The advanced scheme}

In this section we propose the advanced scheme that is more sophisticated than the simple scheme but can offer more accurate fan-out estimations. It is based on the aforementioned design methodology of separating identity gathering from counting. Its system model is shown in Figure 3. There are two parallel modules processing the incoming packet stream. The data streaming module encodes the fan-out information for each and every source (arc 1 in Figure 3 ) into a very compact data structure, and the identity sampling module captures the candidate source identities which have potential to be super sources (arc 2). These source identities are then used by an estimation algorithm to look up the data structure (arc 3) produced by the data streaming module (arc 4) to get their corresponding fan-out estimates. The design of these modules are described in the following subsections.

\subsection{Online streaming module}

The data structure used in the online streaming module is quite simple: an $m \times n 2$-dimensional bit array $A$. The bits

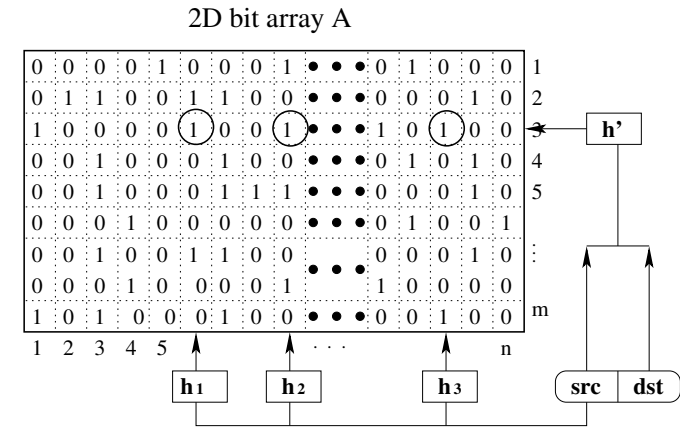

Figure 4: An instance of online streaming module

\begin{tabular}{|l|}
\hline 1. Initialize \\
2. $\quad A[i, j]:=0, \quad i=1,2, \ldots, m \quad j=1,2, \cdots, n$ \\
3. Update \\
4. $\quad$ Upon the arrival of a packet $p k t$ \\
5. $\quad$ row $:=h^{\prime}(<$ pkt.src, pkt.dst $>)$ \\
6. for $i:=1$ to $k$ \\
7. $\quad$ col $:=h_{i}($ pkt.src $)$ \\
8. $\quad A[$ row, col $]:=1$ \\
\end{tabular}

Figure 5: Algorithm of online streaming module

in $A$ are set to all " 0 "s at the beginning of each measurement epoch. The algorithm of updating $A$ is shown in Figure 5. Upon the arrival of a packet pkt, pkt.src is hashed by $k$ independent ${ }^{9}$ hash functions $h_{1}, h_{2}, \cdots, h_{k}$ with range [1..n]. The hashing results $h_{1}$ (pkt.src), $h_{2}$ (pkt.src), ..., $h_{k}$ (pkt.src) are viewed as column indices into $A$. In our scheme, $k$ is set to 3 , and the rationale behind it will be discussed in Section 4.3. Then, the flow label $<$ pkt.src,pkt.dst $>$ is hashed by another independent hash function $h^{\prime}$ (with range $[1 . . m]$ ) to generate a row in$\operatorname{dex}$ of $A$. Finally, the $k$ bits located at the intersections of the selected row and columns are set to "1". An example is shown in Figure 4, in which the three intersection bits (circled) are set to " 1 "s. When $A$ is filled (by " 1 ") to a threshold percentage we terminate the current measurement epoch and start the decoding process ${ }^{10}$. In Section 4.2 , we show that the above process produces a very compact and accurate (statistical) encoding of the fan-outs of the sources, and present the corresponding decoding algorithm.

Readers may feel that the above 2D bit array $A$ is a variant of Bloom filters [1]. This is not the case since although its encoding algorithm is similar to that of a Bloom filter (flipping some bits to " 1 "s), we decode the $2 \mathrm{D}$ bit array for a different kind of information (the fan-out count) than if we really use it as a Bloom filter (check if a particular source-destination pair has appeared). Our encoding algo- 
rithm is not well engineered for being used as a Bloom filter. And our decoding algorithm, shown next, is also fundamentally different from that of a Bloom filter.

The proposed online streaming module has very low memory consumption and high streaming speed:

Memory (SRAM) consumption. Our scheme is extremely memory-efficient. Each source-destination pair (flow) will set 3 bits in the bit vectors to " 1 "s and consume a little more than 3 bits of SRAM storage ${ }^{11}$. We will show that the scheme provides very high accuracy using reasonable amount of SRAM (e.g., 128KB) in Section 6.

Streaming speed. Each update requires only 4 hash function computations and 3 writes to the SRAM. We require that these four hash functions are independent and amendable to hardware implementation. They can be chosen from the $H_{3}$ hash function family [2, 18], which, with hardware implementation, can produce a hash output within a few nanoseconds. Then with commodity $5 n s$ SRAM our scheme would allow around 40 million packets per second, thereby supporting 40 Gbps traffic stream assuming a conservative average packet size of 1,000 bits.

\subsection{Estimation module}

For each source identity recorded by the sampling module (described later), the estimation module decodes its approximate fan-out from the $2 \mathrm{D}$ bit array $A$, the output of the data streaming module. In this section, we describe this decoding algorithm in detail.

When we would like to know $F_{s}$, the fan-out of the source $s, s$ is hashed by the hash functions $h_{1}, \cdots, h_{k}$, which are defined and used in the online streaming module, to obtain $k$ column indices. Let $A_{i}, i=1,2, \cdots, k$, be the corresponding columns (viewed as bit vectors). In the following, we derive, step by step, an accurate and almost unbiased estimator of $F_{s}$, as a function of $A_{i}, i=1,2, \cdots$, $k$.

Let the set of packets hashed into column $A_{i}$ during the corresponding measurement epoch be $T_{i}$ and the number of bits in $A_{i}$ that are " 0 "s be $U_{T_{i}}$. Note that the value $U_{T_{i}}$ is a part of our observation since we can obtain $U_{T_{i}}$ from $A_{i}$ through simple counting, although the notation itself contains $T_{i}$, the size of which we would like to estimate. Recall the size of the column vector is $m$. A fairly accurate estimator of $\left|T_{i}\right|$, the number of packets of $T_{i}$, adapted from [21], is

$$
D_{T_{i}}=m \ln \frac{m}{U_{T_{i}}}
$$

Note that $F_{s}$, the fan-out of the source $s$, is equal to $\mid T_{1} \cap$ $T_{2} \cap \cdots \cap T_{k} \mid$, if during the measurement epoch, no other sources are hashed to the same $k$ columns $A_{1}, A_{2}, \cdots, A_{k}$. Otherwise $\left|T_{1} \cap T_{2} \cap \cdots \cap T_{k}\right|$ is the sum of the fanouts of all (more than 1) the sources that are hashed into $A_{1}, A_{2}, \cdots, A_{k}$. We show in the next section, that the probability with which the latter case happens is very small when $k=3$. We obtain the following estimator of $F_{s}$, which is in fact derived as an estimator for $\mid T_{1} \cap T_{2} \cap \cdots \cap$ $T_{k} \mid$.

$$
\begin{aligned}
\widehat{F_{s}}= & \sum_{1 \leq i \leq k} D_{T_{i}}-\sum_{1 \leq i_{1}<i_{2} \leq k} D_{T_{i_{1}} \cup T_{i_{2}}} \\
& +\sum_{1 \leq i_{1}<i_{2}<i_{3} \leq k} D_{T_{i_{1}} \cup T_{i_{2}} \cup T_{i_{3}}} \\
& +\cdots+(-1)^{k-1} D_{T_{1} \cup T_{2} \cdots \cup T_{k}}
\end{aligned}
$$

Here $D_{T_{i} \cup \cdots \cup T_{j}}$, is defined as $m \ln \frac{m}{U_{T_{i} \cup \cdots \cup T_{j}}}$, where $U_{T_{i} \cup \ldots \cup T_{j}}$ denotes the number of " 0 "s in the bit vector $B_{T_{i} \cup \ldots \cup T_{j}}$ which is the result of hashing the set of packets $T_{i} \cup \cdots \cup T_{j}$ into a single empty bit vector. The bit vector $B_{T_{i} \cup \ldots \cup T_{j}}$ is computed as the bitwise-OR of $A_{i}, \ldots$, $A_{j}$. One can easily verify the correctness of this computation with respect to the semantics of $B_{T_{i} \cup \ldots \cup T_{j}}$.

We need to show that the RHS of Formula 4 is a fairly good estimator of $\left|T_{1} \cap T_{2} \cap \cdots \cap T_{k}\right|$. Note that

$$
\begin{aligned}
& \left|T_{1} \cap T_{2} \cap \cdots \cap T_{k}\right|=\sum_{1 \leq i \leq k} T_{i}-\sum_{1 \leq i_{1}<i_{2} \leq k} T_{i_{1}} \cup T_{i_{2}} \\
& +\sum_{1 \leq i_{1}<i_{2}<i_{3} \leq k} T_{i_{1}} \cup T_{i_{2}} \cup T_{i_{3}} \\
& +\cdots+(-1)^{k-1} T_{1} \cup T_{2} \cdots \cup T_{k}
\end{aligned}
$$

by the principle of inclusion and exclusion. Since $D_{T_{i} \cup \cdots \cup T_{j}}$ is a fairly good estimator of $\left|T_{i} \cup \cdots \cup T_{j}\right|$ according to [21], we obtain the RHS of Formula 4 by replacing the terms $\left|T_{i} \cup \cdots \cup T_{j}\right|$ in Formula 5 by $D_{T_{i} \cup \cdots \cup T_{j}}$, $1 \leq i<j \leq k$. Note that it is not correct to directly use the bitwise-AND of $A_{1}, A_{2}, \ldots, A_{k}$ for estimating $\left|T_{1} \cap T_{2} \cap \cdots \cap T_{k}\right|$ using Formula 3 , because the bit vector corresponding to the result of hashing the set of packets $T_{1} \cap T_{2} \cap \ldots \cap T_{k}$ into an empty bit vector, is not equivalent to the bitwise-AND of $A_{1}, \ldots, A_{k}$.

The estimator in Formula 4 generalizes the result in [21] which is developed for the special case $k=2$. We will show that our scheme only needs to use the special case of $k=3$, which is

$$
\begin{aligned}
\widehat{F_{s}}= & D_{T_{1}}+D_{T_{2}}+D_{T_{3}}-D_{T_{1} \cup T_{2}}-D_{T_{1} \cup T_{3}}-D_{T_{2} \cup T_{3}} \\
& +D_{T_{1} \cup T_{2} \cup T_{3}}
\end{aligned}
$$

The computational complexity of estimating the fan-out of a source is dominated by $2^{k}-k-1$ bitwise-OR operations among $k$ column vectors. Such vectors can be encoded as one or more unsigned integers so that the bitparallelism can significantly reduce the execution time. Since $m$ is typically 64 bits in our scheme, the whole vector can be held in two 32-bit integers. Therefore, in our scheme where $k=3$, estimation of the fan-out of each 
source only needs 8 bitwise-OR operations between 32-bit integers. We also need to count the number of " 0 "s in a vector (to get $U_{T}$ values). This can be sped up significantly by using a pre-computed table (in SRAM) of size 262,144 $\left(=2^{16} \times 4\right)$ bits that stores the number of "0"s in all 16-bit numbers. Our estimation of the execution time shows that our scheme is fast enough to support OC-768 operations.

\subsection{Accuracy analysis}

In this section we first briefly explain the rationale behind setting $k$ to 3 in the estimator and then analyze the accuracy of our estimator rigorously. We set $k$ (the number of "column" hash functions) to 3 due to the following two considerations. First, we mentioned before that if two sources $s_{1}$ and $s_{2}$ both are hashed to the same $k$ columns, our decoding algorithm will give us an estimate of their total fan-out, when we use $s_{1}$ or $s_{2}$ to lookup the 2D array. We certainly would like the probability with which this scenario occurs to be as small as possible. This can be achieved by making $k$ as large as possible. However, larger $k$ implies larger computational and storage complexities at the online streaming module. We will show that $k=3$ makes the probability of the aforementioned hash collision very small, and at the same time keeps the computational and storage complexities of our scheme modest.

Now we derive $\eta$, the probability that at least two sources happen to hash to the same set of $k$ columns by $h_{1}, h_{2}, \ldots$, $h_{k}$. It is not hard to show, using straightforward combinatorial arguments, that $\eta=1-\frac{\left(\begin{array}{c}n^{k} \\ S\end{array}\right) S !}{n^{k S}}$, where $S$ is the total number of the distinct sources during the measurement epoch. We observe that, given typical values for $n$ and $S, \eta$ is quite large when $k=2$, but drops to a very low value when $k=3$. For example, when $n=16 \mathrm{~K}$ and $S=100,000, \eta$ is close to 1 when $k=2$, but drops to 0.002 when $k=3$.

The following theorem characterizes the variance of the estimator in Formula 6, which is also its approximate mean square error (MSE), since the estimator is almost unbiased and the impact of $\eta$ (discussed above) on the estimation error is very small when $k=3$. Its proof can be found in [24]. This is an extension of our previous variance analysis in [23] which is derived for the special case $k=2$. Let $t_{T}$ denote $|T| / m$, which is the load factor of the bit vector(of size $m$ ) when the corresponding set $T$ of source-destination pairs are hashed into it, in the following theorem.

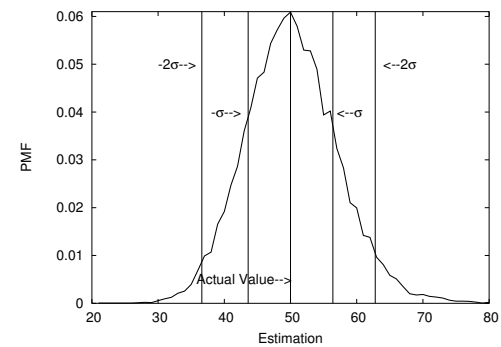

Figure 6: Distribution of the estimation from Monte-Carlo simulation ( $m=64 \mathrm{~b}, k=3$ and $\left.F_{s}=50\right)$. $\sigma$ is the standard deviation computed from Theorem 3

Theorem 3 The variance of $\widehat{F_{s}}$ is given by

$$
\begin{aligned}
& \operatorname{Var}\left[\widehat{F_{s}}\right] \approx-m \sum_{i=1}^{3} f\left(t_{T_{i}}\right)-m \sum_{1 \leq i_{1}<i_{2} \leq 3} f\left(t_{T_{i_{1}} \cup T_{i_{2}}}\right) \\
& +2 m\left(f\left(t_{T_{1} \cup\left(T_{2} \cap T_{3}\right)}\right)+f\left(t_{T_{2} \cup\left(T_{1} \cap T_{3}\right)}\right)+f\left(t_{T_{3} \cup\left(T_{2} \cap T_{1}\right)}\right)\right) \\
& +2 m \sum_{1 \leq i_{1}<i_{2} \leq 3} f\left(t_{T_{i_{1}} \cap T_{i_{2}}}\right) \\
& \quad-2 m\left(f\left(t_{T_{1} \cap\left(T_{2} \cup T_{3}\right)}\right)+f\left(t_{T_{2} \cap\left(T_{1} \cup T_{3}\right)}\right)+f\left(t_{T_{3} \cap\left(T_{2} \cup T_{1}\right)}\right)\right) \\
& +m f\left(t_{T_{1} \cup T_{2} \cup T_{3}}\right) \\
& \text { where } f(t)=e^{t}-t-1 .
\end{aligned}
$$

An example distribution of $\widehat{F_{s}}$ with respect to the actual value $F_{s}$ is shown in Figure 6. We obtained this distribution with 20,000 runs of the Monte-Carlo simulations with the following parameters. In this empirical distribution, the actual fan-out $F_{s}$ is 50, the size of the column vector $m$ is 64 bits. The load factor is set to $t_{T_{i}}=1.5$ for $1 \leq i \leq 3$. Also, since the sets $T_{1}, T_{2}$, and $T_{3}$ have 50 flows in common, we set $t_{T_{i} \cap T_{j}}=\frac{50}{64}=0.78125$, for $1 \leq i<j \leq 3$. Here we implicitly assume that pairs of them do not have any flows in common other than these 50 flows, which happens with very high probability $\left(=1-\frac{1}{n^{2}}+\frac{1}{n^{3}}\right)$ anyway given a large $n$ (e.g., 16K). In this example the standard deviation $\sigma$ is around 6.4 as computed from Theorem 3 . We observe that the size of the tail that falls outside 2 standard deviations from the mean is very small $(<4 \%)$. This shows that, with high probability, our estimator will not deviate too much from the actual value. In Section 6, the trace-driven experiments on the real-world Internet traffic will further validate this. $^{12}$

Note that given the size of $m$, our scheme is only able to accurately estimate fan-out values up to $m \ln m+O(m)$, because if the actual fan-out $F_{s}$ is much larger than that, we will see all 1's in the corresponding column vectors with high probability (due to the result of the "coupon collector's problem" [13]). In this case, the only information we can obtain about $F_{s}$ is that it is no smaller than $m \ln m$. Fortunately, for the purpose of detecting super sources, this information is good enough for us to declare $s$ a super source, 
as long as the threshold for super sources is much smaller than $m \ln m$. However, in some applications (e.g., estimating the spreading speed of a worm), we may also want to know the approximate fan-out value. This can be achieved using a multi-resolution extension of our advanced scheme. The methodology of multi-resolution is quite standard and has been used in several recent works $[6,12,11]$. The extension in our context is straightforward. We omit its detailed specifications here in interest of space.

\subsection{Identity sampling module}

The purpose of this module is to capture the identities of potential super sources that will be used to look up the 2D array to get their fan-out estimations. The filtering after sampling technique proposed in Section 3 is adopted here with a slightly different recording strategy. Instead of constructing a hash table to record the sources and their fan-out estimation, here we only record the source identities sequentially in the DRAM. Since this strategy avoids expensive hash table operations and sequential writes to DRAM can be made very fast (using burst mode), very high sampling rate can be achieved. With commodity $5 \mathrm{~ns}$ SRAM and 60ns DRAM, this recording strategy will be able to process more than 12.5 million packets per second. At this speed, we can record $100 \%$ and $25 \%$ flow labels for OC-192 and OC-768 links respectively. With such a high sampling rate, the probability that the identity of a real super source misses sampling is very low. For example, given $25 \%$ sampling rate the probability that a source with fan-out 50 fails to be recorded is only $5.6 \times 10^{-7}$ $\left(=(1-25 \%)^{50}\right)$.

\section{Estimating outstanding fan-outs}

In this section we describe how to extend the advanced scheme to detect the sources that have contacted but have not obtained acknowledgments from a large number of distinct destinations (i.e., the sources with large outstanding fan-outs). Although both of our schemes have the potential to support this extension we focus on the advanced scheme in this work and leave the extension of the simple scheme for future research. In the following sections we show how to slightly modify the operations of the online streaming module and the estimation module of the advanced scheme for estimating outstanding fan-outs. The sampling module does not need to be modified.

\subsection{Online streaming module}

The online streaming module employs two 2D bit arrays $A$ and $B$ of identical size and shape. The array $A$ encodes the fan-outs of sources in traffic in one direction (called "outbound") in the same way as in the advanced scheme (shown in Figure 5). The array $B$ encodes the fan-ins of the destinations of the acknowledgment packets in the opposite direction (called "inbound"). Its encoding algo-

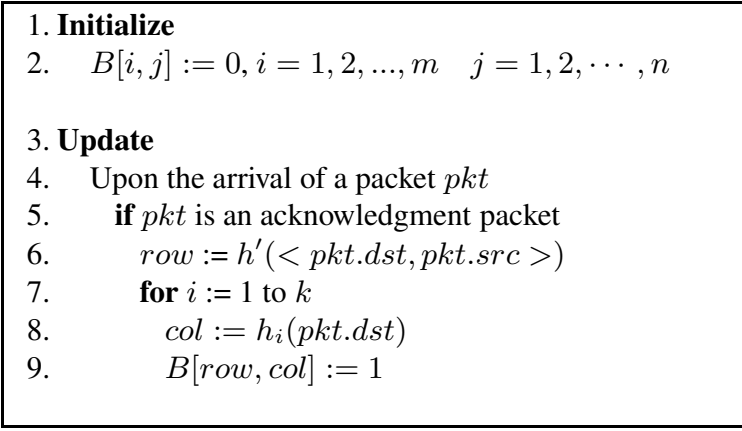

Figure 7: Algorithm for updating the 2D bit array $B$ to record ACK packets

rithm is shown in Figure 7. It is a transposed version of the algorithm shown in Figure 5 in the sense that all occurrences of "pkt.src" are replaced with with "pkt.dst" and "pkt.dst" with "pkt.src". This transposition is needed since a source in the outbound traffic appears in the inbound acknowledgment traffic as a destination, and after transposing two packets that belong to a flow and its "acknowledgment flow" respectively will result in a write of " 1 " to the same bit locations in $A$ and $B$ respectively. This allows us to essentially take a "difference" between $A$ and $B$ to obtain the decoding of outstanding fan-outs of various sources, shown next.

\subsection{Estimation module}

We compute the bitwise-OR of $A$ and $B$, denoted as $A \vee B$. For each source $s$, we decode its fan-out from $A \vee B$ using the same decoding algorithm as described in Section 4.2. Similarly, we decode its fan-in in the acknowledgment traffic from $B$. Our estimator of the outstanding fan-out of $s$ is simply the former subtracted by the latter.

Now we explain why this estimator will provide an accurate estimate of the outstanding fan-out of a source $s$. Let $S_{1}$ be the set of flows whose source is " $s$ " in the outbound traffic. Let $S_{2}$ be the set of flows whose destination is " $s$ " in the inbound acknowledgment traffic. Clearly the quantity we would like to estimate is simply $\left|S_{1}-S_{2}\right|$. The correctness of our estimator is evident from the following three facts: (a) $\left|S_{1}-S_{2}\right|$ is equal to $\left|S_{1} \cup S_{2}\right|-\left|S_{2}\right|$; (b) decoding from $A \vee B$ will result in a fairly accurate estimate of $\left|S_{1} \bigcup S_{2}\right|$ and (c) decoding from $B$ will result in a fairly accurate estimate of $\left|S_{2}\right|$.

\section{Evaluation}

In this section, we evaluate the proposed schemes using real-world Internet traffic traces. Our experiments are grouped into three parts corresponding to the three algorithms presented: the simple scheme, the advanced scheme, and its extension to estimate outstanding fan-outs. The experimental results show that our schemes allow for accu- 


\begin{tabular}{|c|c|c|c|}
\hline Trace & \# of sources & \# of flows & \# of packets \\
\hline \hline IPKS+ & 119,444 & 151,260 & $1,459,394$ \\
\hline IPKS - & 96,330 & 125,126 & $1,655,992$ \\
\hline USC & 84,880 & 106,626 & $1,500,000$ \\
\hline UNC & 55,111 & 101,398 & $1,495,701$ \\
\hline
\end{tabular}

Table 1: Traces used in our evaluation. Note that source is $<$ $s r c_{-} I P, s r c_{-}$port $>$, destination is dst_IP and flow label is the distinct source-destination pair.

rate estimation of fan-outs and hence the precise detection of super sources.

\subsection{Traffic Traces and Flow definitions}

Trying to make the experimental data as representative as possible, we use packet header traces gathered at three different locations of the Internet, namely, University of North Carolina (UNC), University of Southern California (USC), and NLANR. The trace form UNC was collected on a 1 Gbps access link connecting the campus to the rest of the Internet, on Thursday, April 24, 2003 at 11:00 am. The trace from USC was collected at their Los Nettos tracing facility on Feb. 2, 2004. We also use a pair of unidirectional traces from NLANR: IPKS+ and IPKS-, collected simultaneously on both directions of an OC192c link on June 1, 2004. The link connects Indianapolis (IPLS) to Kansas City (KSCY) using Packet-over-SONET. This pair of traces is especially valuable to evaluate the extended advanced scheme for estimating outstanding fan-outs. All the above traces are either publicly available or available for research purposes upon request. Table 1 summarizes all the traces used in the evaluation. We will use $U S C$, $U N C$ and $I P K S+$ to evaluate our simple scheme and advanced scheme and use the concurrent traces $I P K S+$ and $I P K S$ - to evaluate the extension.

As mentioned before, a source/destination label can be any combination of source/destination fields from the IP header. Two different definitions of source and destination labels are used in our experiments, targeting different applications. In the first definition, source label is the tuple $<$ src_IP, src_port $>$ and destination label is $<$ dst_IP $>$. This definition targets applications such as detecting worm propagation and locating popular web servers. The flow statistics displayed in Table 1 use this definition. In the second definition, Second, source label is $\langle$ src IP $>$ and destination label is the tuple $<$ dst_IP, dst_port $>$. This definition targets applications such as detecting infected sources that conduct port scans. The experimental results presented in this section use the first definition of source and destination labels unless noted otherwise.

\subsection{Accuracy of the simple scheme}

In this section, we evaluate the accuracy of the simple scheme in estimating the fan-outs of sources and in detect- ing super sources. Figure 8 compares the fan-outs of the sources estimated using our simple scheme with the their actual fan-outs in traces $I P K S+U N C$, and $U S C$ respectively. In these experiments, a flow sampling rate of $1 / 4$ and a bit array of size $128 \mathrm{~K}$ bits is used. The figure only plots the points whose actual fan-out values are above 15 since lower values (i.e., $<15$ ) are not interesting for finding super sources. The solid diagonal line in each figure denotes perfect estimation, while the dashed lines denote an estimation error of $\pm 15 \%$. The dashed lines are parallel to the diagonal line since both $\mathrm{x}$-axis and $\mathrm{y}$-axis are on the log scale. Clearly the closer the points cluster around the diagonal, the more accurate the estimation is. We observe that the simple scheme achieves reasonable accuracy for relatively large fan-outs in all three traces. Figure 8 also reflects the false positives and negatives in detecting super sources. For a given threshold 50, the points that fall in "Area I" corresponds to false positives, i.e., the sources whose actual fan-outs are less than the threshold but the estimated fan-outs are larger than the threshold. Similarly, the points that fall in "Area II" corresponds to false negatives, i.e., the sources whose actual fan-outs are larger than the threshold but the estimated fan-outs are smaller than the threshold. We observe that in Figure 8, points rarely fall into Areas I and II (i.e., very few false positives and negatives ${ }^{13}$ ).

While this scheme works well with $1 / 4$ sampling rate, it cannot produce good estimations with smaller sampling rates (e.g., 1/16. We omit the experimental results here due to lack of space.). However such lower sampling rates might be necessary to keep up with very high link speeds such as 40 Gbps (OC-768).

We repeat the above experiment under the aforementioned second definition of source and destination, in which the source label is $<$ src_IP $>$ and destination label is $<$ dst_IP, dst_port $>$. Figure 9 plots the estimated fan-outs of sources in trace IPKS+. With this definition the trace $I P K S+$ has 9,359 sources and 140,140 distinct sourcedestination pairs. We can see from the figure that our estimation is also quite accurate with this second definition of source and destination.

\subsection{Accuracy of the advanced scheme}

In this section we evaluate the accuracy of the advanced scheme using both trace-driven simulation and theoretical analysis. The estimation accuracy of the advanced scheme is a function of the various design parameters, including the size and shape of the 2D bit array $A$ (i.e., the number of rows $m$ and columns $n$ ) and the number of hash functions (k).

In the experiments we set the size of $A$ to $128 \mathrm{~KB}$ (64 rows $\times 16,384$ columns), $k=3$ and the flow sampling rate to 1 . This configuration is very space-efficient. For example it only uses 7 bits per flow on the average for the 


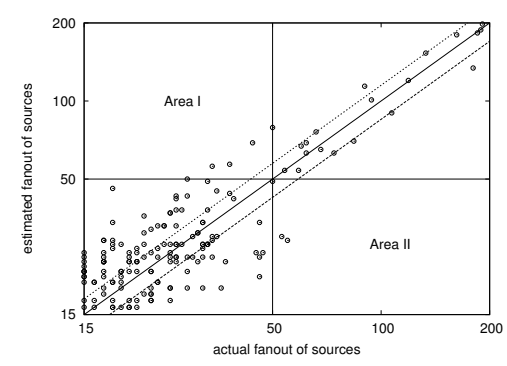

(a) trace IPKS+

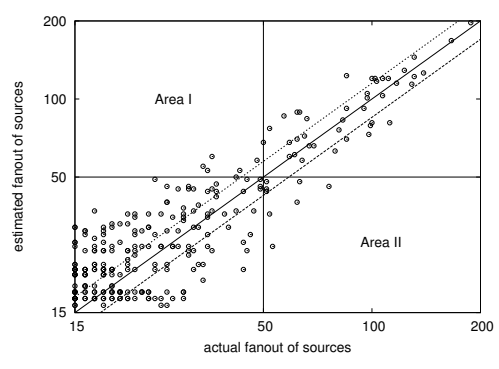

(b) trace UNC

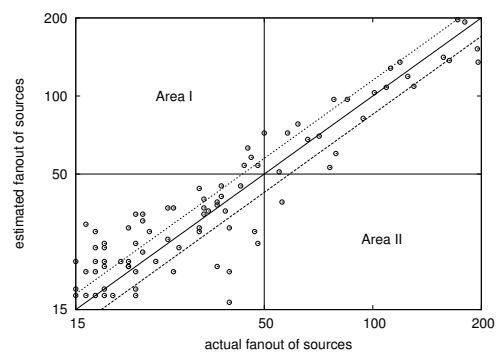

(c) trace USC

Figure 8: Actual vs. estimated fan-outs of sources by the simple scheme given the flow sampling rate 1/4. Notice both axes are on logscale.

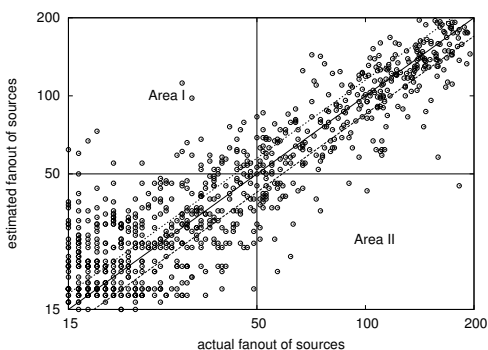

Figure 9: Actual vs. estimated fan-out of sources for trace $I P K S+$ with flow sampling rate $1 / 4$. The aforementioned second definition of source and destination labels is used here. Note that both $\mathrm{x}$-axis and $\mathrm{y}$-axis are on logscale.

trace $I P K S+$.

\subsubsection{Trace-driven experiments}

Figure 10 compares the fan-out values estimated using the advanced scheme with the actual fan-outs of the corresponding sources given three different traces. Compared with the corresponding plots in Figure 8, the points are much closer to the diagonal lines, which means that the advanced scheme is much more accurate than the simple scheme.

In Figure 11, we repeat the experiments with source and destination labels defined as $\langle$ src_IP $\rangle$ and $<$ dst_IP,dst_port $>$, respectively. Compared with the result of the simple scheme (Figure 9) the points are much closer to the diagonal again, indicating the higher accuracy achieved by the advanced scheme.

Note that in the experiments above we set the flow sampling rate to 1 instead of $1 / 4$ used in the experiments of the simple scheme since as we described in Section 3.3 and Section 4.4 respectively for a fully utilized OC- 192 link the simple scheme requires $1 / 4$ flow sampling rate but the identity sampling module of the advanced scheme can record $100 \%$ flow labels.

\subsubsection{Theoretical accuracy}

The accuracy of the estimation can be characterized by the average relative error of the estimator, which is equal to the standard deviation of the ratio $\frac{\widehat{F_{s}}}{F_{s}}$ which can be computed by Theorem 3 .

Figure 12 shows the average relative error plotted against estimated fan-outs for the sources in the trace IPKS+. Experiments on other traces produced similar results. The average relative error shows a sharply downward trend when the estimated value of fan-out increases in Figure 12. This is a very desirable property as we would like our mechanism to be more accurate when estimating larger fanouts. Towards the right extreme of the figure, the average relative error starts to increase. This is because the selected bit vectors become almost full ("saturation") when the fanout value is close to $266(\mathrm{~m} \ln m)$. As we discussed in Section 4.3 the accuracy of our estimator would degrade when the corresponding column vectors become saturated ${ }^{14}$. It does not affect the accuracy of our scheme for detecting super sources, but to accurately estimate the exact fan-out values that are large, the aforementioned multi-resolution extension $[6,11,12]$ is needed.

The accuracy of the estimator can also be characterized by the probability of the estimated values $\widehat{F_{s}}$ falling into the interval $\left[(1-\epsilon) F_{s},(1+\epsilon) F_{s}\right]$, where $F_{s}$ is actual fan-out of the source $s$. This quantity can be numerically computed by Monte-Carlo Simulation as follows. We first use the trace $U N C$ to construct the $2 \mathrm{D}$ bit array $A$ (serving as "background noise"). Then we synthetically generate a source that has fan-out value $F_{s}$ and insert it into $A$ by randomly selecting 3 different columns. The estimator (Formula 6 ) is used to obtain the $\widehat{F_{s}}$. The above operations are repeated 100,000 times to compute the probabilities shown in Figure 12 .

Figure 13 shows the plot of $(1-\delta)$ for different values of $F_{s}$, where $1-\delta=\operatorname{Prob}\left[(1-\epsilon) F_{s} \leq \widehat{F_{s}} \leq(1+\right.$ $\left.\epsilon) F_{s}\right]$. Each curve corresponds to a specific level of relative 


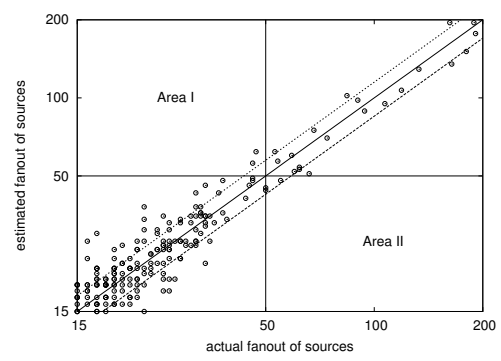

(a) trace IPKS+

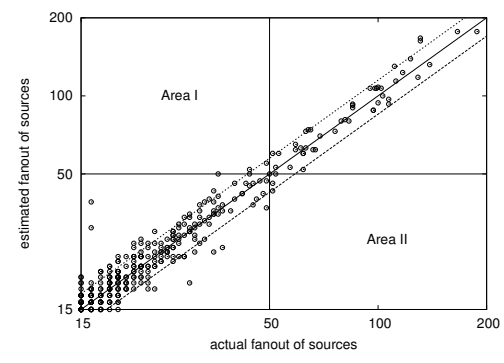

(b) trace UNC

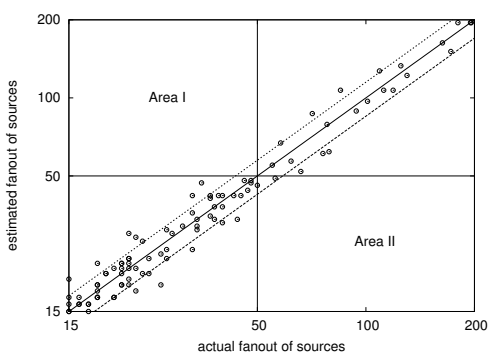

(c) trace USC

Figure 10: Actual vs. estimated fan-out of sources by the advanced scheme. Notice both axes are on logscale.
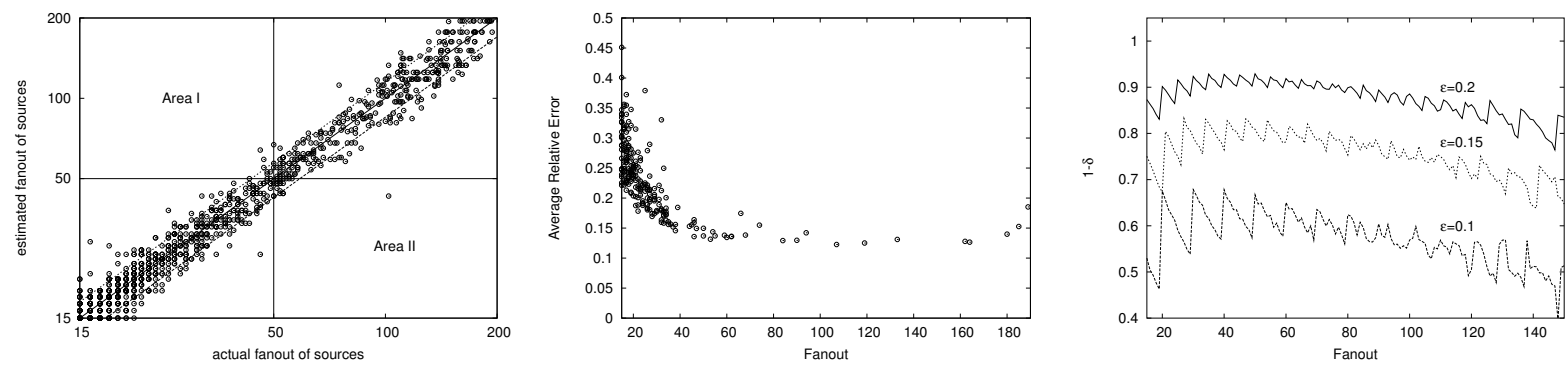

Figure 11: Actual vs. estimated fan-out Figure 12: Average relative error for vari- Figure 13: Probability that the estimate of sources for trace $I P K S+$ under the ous fan-out values in the trace $I P K S+. \widehat{F_{S}}$ is within a factor of $(1 \pm \epsilon)$ of the actual second flow definition by the advanced fan-out $F_{s}$ for various values of $\epsilon$.

scheme. Notice both axes are on logscale.

error tolerance, i.e., a specific choice of $\epsilon$, and represents the probability that the estimated value is within this factor of the actual value. For example, the curve for $\epsilon=0.2$ shows that around $85 \%$ of the time the estimate is within $20 \%$ of the actual value. Notice how the curves in the figure have an upward trend first and then show a downward trend as the fan-out increases further. This corresponds exactly to the aforementioned "saturation" situation.

\subsection{Accuracy of the extension to estimate outstanding fan-outs}

To evaluate the extension of the advanced scheme to estimate outstanding fan-outs we use the pair of traces, $I P K S+$ and $I P K S-$, collected simultaneously on both directions of a link. We extract all the acknowledgment packets from $I P K S$ - to produce the $2 \mathrm{D}$ bit array $B$ using the transposed update algorithm (Figure 7). The same parameters are configured for both $2 \mathrm{D}$ bit arrays $A$ and $B$. Figure 14 shows the scatter diagram of the fan-out estimated using our proposed scheme ( $y$ axis) vs. actual outstanding fan-out ( $x$ axis). The fact that most points are concentrated within a narrow band of fixed width along the diagonal line indicates that our estimator is accurate on estimating outstanding fan-outs.

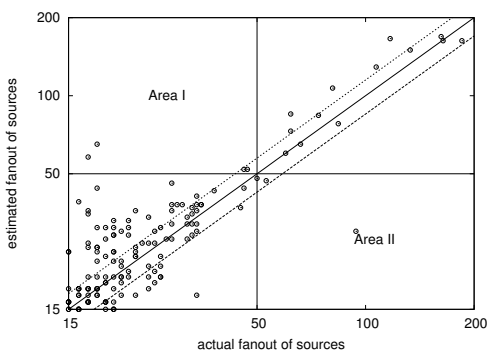

Figure 14: Actual vs. estimated fan-out of sources by extension of the advanced scheme including deletions. Notice both axes are on logscale.

\section{Related work}

The problem of detecting super sources and destinations has been studied in recent years. In general, three approaches have been proposed in the literature:

1. A straightforward approach is to keep track, for each source/destination, the set of distinct destinations/sources that it contacts, using a hash table. This approach is adopted in Snort [19] and FlowScan [17]. It is straightforward to implement but not memory-efficient, since most of the source-destination pairs in the hash table do not come 
from super sources/destinations. As mentioned before, this approach is not feasible for monitoring high-speed links since the hash table typically can only fit into DRAM.

2. Data streaming algorithms are designed by Estan et al. [6] mainly for estimating the number of active flows in the Internet traffic. However, it is stated in [6], that one variant of their scheme, i.e., triggered bitmap, can be used for identifying the super sources. This algorithm maintains a small bitmap (4 bytes) for each source (subject to hash collision), for estimating its fan-out. Once the number of bits set in the small bitmap exceeds a certain threshold (indicting a large fan-out), a large multi-resolution bitmap is allocated to perform a more accurate counting of its fanout. Since the implementation of the binding between the source and the bitmap is not elaborated in [6], we speculate that the binding is implemented as a hash table, which can be quite costly if it has to fit in SRAM (for high-speed processing). Also, its memory efficiency is further limited by allocating at least 4 bytes for each source.

3. Recently Venkataraman et al. [20] propose two flow sampling based techniques for detecting super sources/destinations. Their one-level and two-level filtering schemes both use a traditional hash-based flow sampling technique for estimating fan-outs. We explained in Section 3.1 that, when this scheme is used for high-speed links (e.g., 10 or $40 \mathrm{Gbps}$ ), the sampling rate is typically low due to the aforementioned traffic burst problem. This prevents the algorithms from achieving high estimation accuracy. In addition, the memory usage of both schemes, which use hash tables, is much higher than our advanced scheme. They only mentioned the possibility of replacing hash table with Bloom filters to save space, but did not fully specify the details of the scheme (e.g., parameter settings). This makes a head-on comparison of our schemes with theirs very difficult. In fact, after this replacement (of hash table with Bloom filters), their scheme becomes a variant of Space Code Bloom Filter (SCBF) we proposed in [12], with a slightly different decoding algorithm ${ }^{15}$. Their decoding algorithm has similar computational complexity as that of SCBF, which is an order magnitude more expensive than that of our advanced scheme. This may prevent our SCBF scheme (and their scheme as well) from operating at very high link speeds (e.g., $40 \mathrm{Gbps}$ ).

\section{Conclusion}

Efficient and accurate detection of super sources and destinations at high link speeds is an important problem in many network security and measurement applications. In this work we attack the problem with a new insight that sampling and streaming are often suitable for capturing different and complementary regions of the information spectrum, and a close collaboration between them is an excellent way to recover the complete information. This insight leads to two novel methodologies of combining the power of streaming and sampling, namely, "filtering after sampling" and "separation of counting and identity gathering", upon which our two solutions are built respectively. The first solution improves the estimation accuracy of hashbased flow sampling by allowing for much higher sampling rate, through the use of a embedded data streaming module for filtering/smoothing the bursty incoming traffic. Our second solution combines the power of data streaming in efficiently retaining and estimating fan-out/fan-in associated with a given source/destination, and the power of sampling in generating a list of candidate source/destination identities. Mathematical analysis and trace-driven experiments on real-world Internet traffic show that both solutions allow for accurate detection of super sources and destinations.

\section{References}

[1] B. H. Bloom. Space/time trade-offs in hash coding with allowable errors. CACM, 13(7):422-426, 1970.

[2] J. Carter and M. Wegman. Universal classes of hash functions. Journal of Computer and System Sciences, pages 143-154, 1979.

[3] N. Duffield and M. Grossglauser. Trajectory sampling for direct traffic observation. IEEE transaction of Networking, pages 280-292, June 2001.

[4] N. Duffield, C. Lund, and M. Thorup. Estimating flow distribution from sampled flow statistics. In Proc. ACM SIGCOMM, August 2003.

[5] C. Estan and G. Varghese. New Directions in Traffic Measurement and Accounting. In Proc. ACM SIGCOMM, August 2002.

[6] C. Estan and G. Varghese. Bitmap algorithms for counting active flows on high speed links. In Proc. ACM/SIGCOMM IMC, October 2003.

[7] W. Fang and L. Peterson. Inter-AS traffic patterns and their implications. In Proc. IEEE GLOBECOM, December 1999.

[8] N. Hohn and D. Veitch. Inverting sampled traffic. In Proc. ACM/SIGCOMM IMC, October 2003.

[9] J. Jung, B. Krishnamurthy, and M. Rabinovich. Flash crowds and denial of service attacks: Characterization and implications for cdn and web sites. In Proc. World Wide Web Conference, May 2002.

[10] R. Karp, S. Shenker, and C. Papadimitriou. A simple algorithm for finding frequent elements in streams and bags. ACM Transactions on Database Systems (TODS), 28:51-55, 2003.

[11] A. Kumar, M. Sung, J. Xu, and J. Wang. Data streaming algorithms for efficient and accurate estimation of flow size distribution. In Proc. ACM SIGMETRICS, 2004.

[12] A. Kumar, J. Xu, J. Wang, O. Spatschek, and L. Li. Space-Code Bloom Filter for Efficient per-flow Traffic Measurement. In Proc. IEEE INFOCOM, March 
2004.

[13] R. Motwani and P. Raghavan. Randomized Algorithms. Cambridge University Press, 1995.

[14] CISCO Tech Notes. Cisco netflow. available at http://www.cisco.com/warp/public/732/netflow/ index.html.

[15] V. Paxon. An analysis of using reflectors for distributed denial-of-service attacks. Coтputer Соттиnication Review, 2001.

[16] D. S. Phatak and T. Goff. A novel mechanism for data streaming across multiple IP links for improving throughput and reliability in mobile environments. In Proc. IEEE INFOCOM, June 2002.

[17] D. Plonka. Flowscan: A network traffic flow reporting and visualization tool. In Proc. USENIX LISA, 2000.

[18] M. Ramakrishna, E. Fu, and E. Bahcekapili. Efficient hardware hashing functions for high performance computers. IEEE Transactions on Computers, pages 1378-1381, 1997.

[19] M. Roesch. Snort-lightweight intrusion detection for networks. In Proc. USENIX Systems Administration Conference, 1999.

[20] S. Venkataraman, D. Song, P. Gibbons, and A. Blum. New streaming algorithms for fast detection of superspreaders. In Proc. NDSS, 2005.

[21] K.Y. Whang, B.T. Vander-zanden, and H.M. Taylor. A linear-time probabilistic counting algorithm for database applications. IEEE transaction of Database Systems, pages 208-229, June 1990.

[22] Y. Zhang, S. Singh, S. Sen, N. Duffield, and C. Lund. Online identification of hierarchical heavy hitters: Algorithms, evaluation, and application. In Proc. ACM/SIGCOMM IMC, October 2004.

[23] Q. Zhao, A. Kumar, J. Wang, and J. Xu. Data streaming algorithms for accurate and efficient measurement of traffic and flow matrices. In Proc. ACM SIGMETRICS, June 2005.

[24] Q. Zhao, A. Kumar, and J. Xu. Joint data streaming and sampling techniques for detection of super sources and destinations. In Technical Report, July 2005.

\section{Notes}

1. Super sources have also been referred to as "superspreaders" in literature [20].

2. As a note of clarification, the term data streaming here has no connection with the transmission of multimedia data known as media (audio and video) streaming [16].

3. There is no explicit inversion procedure to recover the number of flows if packet sampling is used. The technique used in [4] may be helpful but does not provide accurate answers.

4. A small buffer in SRAM will not be able to smooth out such bursts since at high link speeds, such bursts can easily fill up several Megabytes of buffer in a matter of milliseconds.

5. We can use multiple independent hash functions to reduce the probability of collisions. But it will significantly increases the overhead of updating $G$ and does not improve the estimation result too much.

6. Note that the worst case for hash-based flow sampling is different. It occurs when a few of the sampled flows contain most of the traffic on a link.

7. The inter-arrival time is in fact of geometric distribution.

8. We assume a conservative average packet size of 1,000 bits, to our disadvantage. Measurements from real-world Internet traffic report much larger packet sizes.

9. Such hash functions are referred to as $k$-universal hash function in literature [2]. It has been shown empirically in [2] that the $H_{3}$ family of hash functions are very close to $k$-universal statistically when operating on real-world data, for small $k$ values (e.g., $k \leq 4$ ).

10. Again, two ping-pong modules can be used in an alternating fashion to avoid any operational interruption.

11. This is estimated based on the typical load factor (defined later) we place on the bit vector.

12. Note that we do not show an example distribution for the previous simple scheme since the estimator $\widehat{F_{s}}$ of it relies on where the flows with source $s$ appear in the packet stream, i.e., the values of $u_{j}$ when the flows arrive (cf. Formula 2). Therefore the estimator may have different distributions given a fixed value of $F_{s}$.

13. One shall not simply compare this false positive and negative ratios with the results in [20] since there only when the scheme fails to detect a source whose fan-out is several (say 5) times larger than the threshold will a false negative be declared.

14. For more details about this please refer to [21].

15. In [12], we decode for the exact value of the parameter to be estimated while their scheme [20] decodes for a lower bound of the parameter. 